\title{
Comparison of antidepressant classes and the risk and time course of suicide attempts in adults: propensity matched, retrospective cohort study
}

Robert J. Valuck, Anne M. Libby, Heather D. Anderson, Richard R. Allen, Indiana Strombom, Lauren B. Marangell and David Perahia

\section{Background}

Placebo-controlled clinical trials have led to concern over possible increased risk of suicide-related events in some populations exposed to antidepressants.

\section{Aims}

To evaluate the risk of suicide attempts by antidepressant drug class and the presence or absence of depression.

\section{Method}

A retrospective propensity-matched new-user cohort study was used to compare participants with incident depression classified by antidepressant treatment with each other and with the general population.

\section{Results}

Among the treated group, the suicide attempt rate peaked in the month prior to diagnosis then decreased steadily over the next 6 months. Among the pharmacologically untreated group, the highest rate was seen in the second month after diagnosis. Cohorts with depression had significantly higher suicide attempt risk than the general population, but the treated group did not differ significantly from the untreated group.

\section{Conclusions}

Patients on antidepressants did not have significantly higher risk compared with untreated patients. No significant differences were observed for patients treated with individual serotonin-noradrenaline reuptake inhibitors (SNRIS) or selective serotonin reuptake inhibitors (SSRIS) or by class (SSRI V. SNRI cohorts).

\section{Declaration of interest}

R.J.V. has received research grants from Eli Lilly and Company. D.P., I.S. and L.B.M. were employees of Eli Lilly and Company at the time this research was conducted.

\section{Copyright and usage}

(c) The Royal college of Psychiatrists 2016.
Since the mid-twentieth century, the question of whether an association exists between antidepressant use and suicide-related events has been the subject of ongoing interest. ${ }^{1}$ Although the initial enquiries were about adult users of antidepressants, the focus expanded early on to include paediatric users. ${ }^{2-4}$ Both the US Food and Drug Administration (FDA) and the European Union European Medicines Agency (EMA) responded with warnings for all or some of these agents, leading to changes in patterns of medical care and use of antidepressants. ${ }^{5-7}$ Currently, specific concerns centre on the possibility of increased risk of suicide-related events with the newer-generation serotoninnoradrenaline reuptake inhibitor (SNRI) antidepressants including venlafaxine and duloxetine, particularly when used by children, adolescents or young adults. ${ }^{8}$ However, the generalisability of previous study results to current medical practice is unclear, as many have been limited by brief trial duration, ${ }^{9-12}$ varying definitions of suicide-related events, lack of statistical power for rare events such as suicide or suicide attempt ${ }^{13-15}$ and inadequate control for non-random allocation, among other issues. ${ }^{16}$ The lack of a community-based estimate of the risk of suicide-related events among people diagnosed with depression, but not treated with antidepressants, has also been an important gap in the literature, as has the lack of an estimate for the baseline rate of such events in people in the community at large (i.e. the general population). This information is critically important to the debate. Without the inclusion of these comparison groups in an analysis, it is not possible to determine the extent to which the risk of suicide-related events is the result of the underlying disease process itself, for example depression, to a possible increase in risk because of the antidepressant treatment in general, or to a possible increase in risk for specific antidepressant drugs or drug classes. The present study, initiated in response to a regulatory request by the EMA, seeks to address these gaps. Specifically, we aim to contribute to the debate in three major ways. First, by relying on data on real-world depression treatment across the USA from 1997 to 2007 to allow a focus on the most commonly used classes of antidepressants (SNRIs, selective serotonin reuptake inhibitors (SSRIs) and tricyclic antidepressants (TCAs)), to establish risk estimates for patients treated with the newest group of antidepressants (SNRIs) relative to the other classes. Second, by creating two distinct populations, an incident depression cohort (both pharmacologically treated and untreated) and a general population cohort against whom suicide attempt risk is compared. Third, by using state of the art epidemiological and statistical methods to account for non-random allocation for treatment and consequent differences in the characteristics of individuals in the different cohorts, using propensity-matched new-user cohorts and multivariate adjustment for known, measured covariates.

\section{Method}

\section{Data source}

This study used data from the IMS (formerly PHARMetrics) LifeLink Database, a commercially available claims database provided by IMS Health. ${ }^{17}$ The LifeLink database is among the largest patient-centric databases of longitudinal, integrated medical and pharmacy claims data in the USA, making it ideal for studies of 
rare outcomes associated with commonly prescribed drug therapies. The integrated data obtained for this study included paid claims from 85 managed care plans nationally - representing 55 million covered lives - from January 1997 to December 2007. Two different data extracts were obtained by the authors. One extract included all records for patients with any mental health diagnosis (ICD-9 code 296.xx - 300.xx or $311 . x x)^{18}$ or an antidepressant prescription dispensed or one or more medically coded suicide attempts; this resulted in approximately 6 million adult patients and was used to create the pharmacologically treated and untreated depression groups. The second extract was a $10 \%$ random sample of the entire LifeLink database, irrespective of diagnosis or treatment; this resulted in approximately 3.5 million adult patients and was used to create a community-based general population sample for comparison. Data were unidentified and anonymous, and the study protocol and data use agreement were approved by the Colorado Multiple Institutional Review Board (COMIRB, Aurora, Colorado, USA).

\section{Study cohorts}

Figure 1 presents a consort chart depicting the creation of the study cohorts. First, a prevalent cohort of participants with current depression was created by identifying patients in the LifeLink mental health extract with a claim for depression (ICD-9 code 296.2x, 296.3x, 300.4x or 311.xx) between 1 August 2004 and 30 June 2007. Patients were excluded if they were younger than 19 years of age (to match FDA age-group specifications) or had less than 6 months of continuous insurance enrolment prior to any depression claim; this selection resulted in a cohort of 1155356 participants age 19 and older with at least one depression claim during the study period. The first depression claim was identified as the participant's index depression diagnosis.

From the prevalent depression cohort, an incident depression cohort was created by excluding patients who had less than 12 months of continuous insurance enrolment prior to an index depression diagnosis or had any of the following during the 12 months prior to their index depression diagnosis: any previous depression diagnosis codes, receipt of any antidepressant or receipt of any psychotherapy services as a paid claim. The time period prior to a depression diagnosis was also used to assess other known risk factors for suicide attempts, such as prior attempts. The resulting incident depression cohort represents participants with 'new' episodes of depression; it should be noted that these episodes are not necessarily 'first-ever' episodes, as the time period used for defining incident episodes was 1 year, and participants may have had episode(s) at some point in the more distant past.

The incident depression cohort $(n=434498)$ was divided into five mutually exclusive treatment groups: those with a dispensed prescription for one of four types of antidepressants (SNRI, SSRI, TCA or multiple antidepressants, defined below) within 30 days of their index depression diagnosis, and those who did not receive antidepressant therapy at all during the study period (untreated depression group). Participants who received an antidepressant other than an SNRI, SSRI or TCA, and participants who received an antidepressant more than 30 days after their index depression diagnosis were excluded $(n=76147)$.

\section{Measures}

Treatment/no treatment groups defined by drug class

Treatment groups were defined on the basis of a dispensed prescription for one of four drug classes within 30 days following the index depression date. The no treatment group was defined as the absence of an antidepressant prescription dispensed of a given type during the study period. SNRIs included duloxetine and venlafaxine and were identified using Medi-Span Electronic Drug File $^{\mathrm{TM}}$ (Med-File) v.2 Generic Product Identifier (GPI) code 5818. SSRIs included citalopram, escitalopram, fluoxetine, fluvoxamine, paroxetine and sertraline and were identified using GPI code 5816. TCAs were identified using GPI code 5820. The multiple antidepressants group was defined as at least one dispensed prescription in a drug class under study within the first 30 days after diagnosis and at least one antidepressant prescription dispensed from another drug class within the 6-month follow-up period. NonSNRI, -SSRI or -TCA antidepressants - namely bupropion, isocarboxazid, maprotiline, mirtazapine, nefazodone, phenelzine, selegiline, tranylcypromine and trazodone - were excluded.

\section{Index date}

For the four antidepressant treatment groups (SNRI, SSRI, TCA or multiple antidepressants), the index date was defined as the date of the first dispensing of the drug in the study period. For the untreated depression group, the index date was defined as the date of their index depression diagnosis. For the general population sample, the index date was defined as the index date for their match from the matched SNRI group.

\section{Suicide attempt}

The primary study outcome was suicide attempt leading to a medical encounter. Suicide attempts leading to medical encounters were identified from all medical or facility claim records by a diagnosis code in the following range (following Centers for Disease Control and Prevention guidelines): ICD-9 codes E950E959 ${ }^{19}$ or ICD-10 codes X60-X84 or Y87.0. ${ }^{20}$ Suicide attempts were considered study outcomes if they occurred at any time during the follow-up period, which started on the index date and lasted for 6 months; or up until the participant had a 1-month gap in continuous insurance enrolment; or had their first suicide attempt. Completed suicides are not captured in the LifeLink database and were thus not included as an outcome measure in this study.

\section{Covariates}

Demographic covariates included gender, region (East, Midwest, South and West), Medicaid status at time of index depression diagnosis and age at index depression diagnosis. Age groups were created based on the following definitions for comparison with FDA suicidality risk communications: young adult, age 19-24 years; adult, age 25-64 years; and older adult, age 65 and older.

Other covariates included both specific and total numbers of mental health comorbidities; specific chronic and acute non-mental health comorbidities; indices of chronic comorbidity including the Chronic Disease Indicator $(\mathrm{CDI})^{21}$ score and the Charlson comorbidity score; ${ }^{22}$ prior medication use (drug-months of exposure to both psychotropic medications and all prescription medications); use of health services; history of suicide attempt; severity of the index depression diagnosis; physician-level covariates; and a market-level covariate reflecting rates of prescribing of each drug group by generalist $v$. specialist prescribers during the month that antidepressant therapy was initiated. Covariates were all assessed during the 6 months prior to the index depression diagnosis and are detailed in Tables 1 and 2.

\section{Statistical analysis}

To account for non-random treatment allocation, we used propensity score matching. This is among the strongest forms of adjustment, balancing measured covariates for the treatment and comparison groups before analysis. ${ }^{23}$ First, the propensity for receiving an SNRI ( $v$. SSRI, TCA, multiple antidepressants 


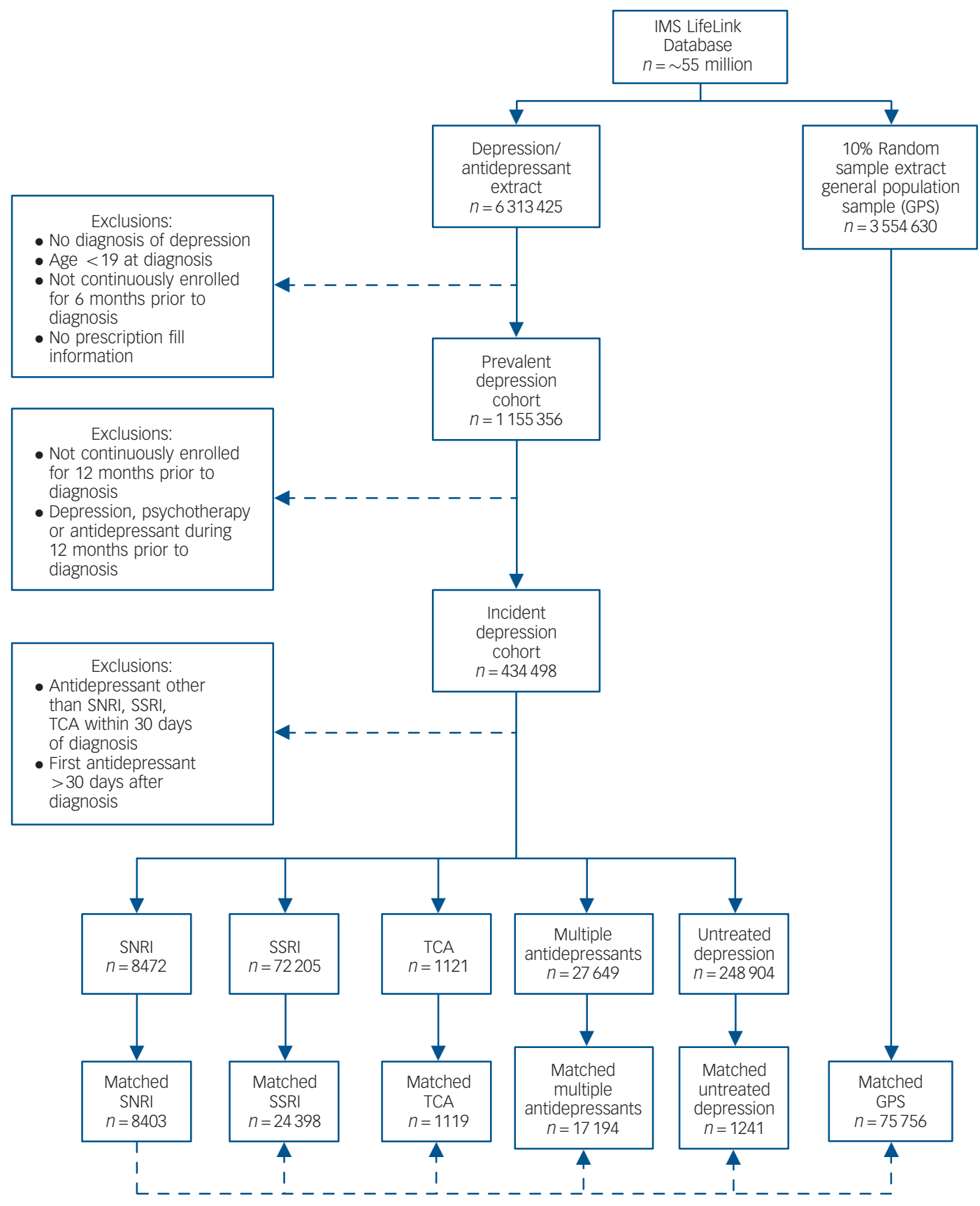

Fig. 1 Flow chart depicting creation of study cohorts.

SNRI, serotonin-noradrenaline reuptake inhibitor; SSRI, selective serotonin reuptake inhibitor; TCA, tricyclic antidepressant.

or no antidepressants) was estimated for each participant in the incident depression cohort using a multivariate unconditional logistic regression model. This model included SNRI treatment (yes/no) as the dependent variable and specified baseline patient-, physician- and market-level measures as covariates. The sets of patient-, physician- and market-level variables were based on prior research and published literature and were assessed for each participant during the 6 months prior to their index depression diagnosis.

Next, participants in the SNRI group were matched to participants from the SSRI, TCA, multiple antidepressants and no antidepressant groups, in a pairwise fashion, using the propensity scores calculated in the previous step. Matching ratios (i.e. the number of participants in the SNRI group matched to those in each comparison group) were 1:3 for each drug-group pair with the exception of TCA, which was matched to the SNRI group with a ratio of 1:1 because of the small sample of adults receiving TCAs as initial therapy. Caliper width (i.e. the allowable amount of deviation in scores among matches) was set at 0.001 for each match. For each pairwise match, if there were more than three (or one for TCA) participants that matched on propensity score within 0.001 , the participants to be matched were chosen randomly from those eligible. This step in the pairwise matching on propensity score resulted in an incident cohort of 52355 participants. In the original SNRI group, 69 participants did not have a match in any of the other drug groups and therefore the size of the matched SNRI group is slightly smaller than the original SNRI group.

The final step in creating the analytic cohort was to match participants from the matched SNRI group $(n=8403)-$ the 
primary group of interest - to participants in the $10 \%$ general population random sample. Participants were matched on age (plus or minus 1 year), gender and region, with up to 10 participants from the general population sample being matched to each participant in the matched SNRI group. If there were more than 10 eligible matches from the general population sample for any participant in the matched SNRI group, 10 general population sample participants were randomly chosen for the match. The resulting analytic cohort included a total of 128111 participants (52355 from the incident depression cohort and 75756 from the general population sample).

Analyses included both the propensity-matched drug-group cohorts and the SNRI-general population sample cohort matched on age, gender and region $(n=128111)$. Analyses were initially stratified by age group (young adult, adult and older adult), then combined and reported in aggregate when similar results were obtained across stratified groups. SAS version 9.1 was used for all data creation and analyses. An alpha-level of 0.05 and 95\% confidence intervals were used to indicate statistical significance.

To identify periods of potentially increased risk of suicide attempt in relation to initiation of antidepressant treatment or no treatment, rates of suicide attempt were aggregated monthly, by antidepressant class, in relation to the patients' index date. Rates are reported per 100000 participants for each month during the 6 months prior to and 6 months following index dates by treatment cohort. For patients in the four treatment groups, monthly incidence rates are anchored by the initiation of antidepressant treatment; for patients in the untreated depression group, incidence rates are anchored by the index depression diagnosis; and for patients in the general population sample, incidence rates are anchored by their SNRI-matched-participant index date. To characterise risk of suicide attempt following initiation of treatment, crude suicide attempt rates during the follow-up period were calculated per 100000 participants for each treatment/no treatment group. Absolute (attributable) risk differences were calculated by subtracting the rate for each depression group (treated and untreated) from the rate for the general population sample.

To estimate a fully adjusted effect of treatment group (SNRI, SSRI, TCA or multiple antidepressants, untreated depression, general population sample) on risk of suicide attempt, multivariate Cox proportional hazards regression models were used, with propensity score included as a covariate, for the outcome days to first suicide attempt. Patients with multiple suicide attempts were censored at the time of first attempt, regardless of how many additional attempts they may have had. Patients with no suicide attempt during the follow-up period were by default censored at the end of follow-up. Demographic characteristics, comorbidities, drug-months of exposure to prior prescriptions (psychotropic and total) and suicide attempt history were included in the models as covariates. Models were run separately by age group and the referent group was varied to estimate the statistical effects compared with the general population sample, untreated depression group or the SNRI group, respectively.

\section{Results}

Descriptive statistics on the matched, incident depression cohort (treated and untreated) and the general population sample are presented in Tables 1 and 2 (online Tables DS1-6 provide descriptive statistics on the cohorts both prior to, and after, propensity

\begin{tabular}{|c|c|c|c|c|c|c|}
\hline & $\begin{array}{l}\text { SNRI group } \\
(n=8403)\end{array}$ & $\begin{array}{l}\text { SSRI group } \\
(n=24398)\end{array}$ & $\begin{array}{l}\text { TCA group } \\
(n=1119)\end{array}$ & $\begin{array}{c}\text { Multiple } \\
\text { antidepressants } \\
\text { group }(n=17 \text { 194) }\end{array}$ & $\begin{array}{c}\text { Untreated } \\
\text { depression group } \\
(n=1241)\end{array}$ & $\begin{array}{c}\text { General population } \\
\text { sample } \\
(n=75756)\end{array}$ \\
\hline Age at index date, years: mean (median) & $41.99(43.00)$ & $42.18(42.00)$ & $44.92(45.00)$ & $41.28(42.00)$ & $44.22(45.00)$ & $41.93(43.00)$ \\
\hline Range, minimum-maximum & 19-98 & 19-98 & 19-98 & 19-98 & 19-98 & 19-98 \\
\hline \multicolumn{7}{|l|}{ Age group, $n(\%)$} \\
\hline Young adult, 19-24 years & $787(9.37)$ & 2675 (10.96) & $66(5.90)$ & $1698(9.88)$ & $106(8.54)$ & 7341 (9.69) \\
\hline Adult, 25-64 years & $7546(89.80)$ & $21051(86.28)$ & $1016(90.80)$ & $15272(88.82)$ & $1094(88.15)$ & $67731(89.41)$ \\
\hline Older adult, $65+$ years & $70(0.83)$ & $672(2.75)$ & $37(3.31)$ & $224(1.30)$ & $41(3.30)$ & $684(0.90)$ \\
\hline Female, $n(\%)$ & $5644(67.17)$ & $16560(67.87)$ & $757(67.65)$ & 11339 (65.95) & 807 (65.03) & 49209 (64.96) \\
\hline Medicaid status, $n$ (\%) & $77(0.92)$ & $276(1.13)$ & $31(2.77)$ & $236(1.37)$ & $25(2.01)$ & $1026(1.35)$ \\
\hline \multicolumn{7}{|l|}{ Region, $n$ (\%) } \\
\hline East & $1893(22.53)$ & $5417(22.20)$ & $237(21.18)$ & $3545(20.62)$ & $361(29.09)$ & $17982(23.74)$ \\
\hline Midwest & $3626(43.15)$ & 10390 (42.59) & $500(44.68)$ & $8227(47.85)$ & $407(32.80)$ & $31371(41.41)$ \\
\hline South & $2298(27.35)$ & $6756(27.69)$ & $261(23.32)$ & $4139(24.07)$ & 369 (29.73) & $20845(27.52)$ \\
\hline West & $586(6.97)$ & $1835(7.52)$ & $121(10.81)$ & $1283(7.46)$ & $104(8.38)$ & $5558(7.34)$ \\
\hline \multicolumn{7}{|l|}{$\begin{array}{l}\text { Suicide attempt history (6 months } \\
\text { prior to index depression date) }\end{array}$} \\
\hline Any previous attempt, $n$ (\%) & $35(0.42)$ & $92(0.38)$ & $2(0.18)$ & $106(0.62)$ & $6(0.48)$ & $10(0.01)$ \\
\hline $\begin{array}{l}\text { Number of previous attempts, } \\
\text { mean (median) }\end{array}$ & $0.00(0.00)$ & $0.00(0.00)$ & $0.00(0.00)$ & $0.01(0.00)$ & $0.00(0.00)$ & $0.00(0.00)$ \\
\hline Range, minimum-maximum & $0-3$ & $0-2$ & $0-1$ & $0-2$ & $0-1$ & $0-1$ \\
\hline $\begin{array}{l}\text { Any attempts resulting in } \\
\text { admission to hospital, } n \text { (\%) }\end{array}$ & $0(0.00)$ & $0(0.00)$ & $0(0.00)$ & $2(0.01)$ & $0(0.00)$ & $2(0.00)$ \\
\hline Prior drug overdose history, ${ }^{a} n(\%)$ & $29(0.35)$ & $73(0.30)$ & $4(0.36)$ & $80(0.47)$ & $14(1.13)$ & $53(0.07)$ \\
\hline \multicolumn{7}{|l|}{$\begin{array}{l}\text { Index depression severity indicators, } \\
n(\%)\end{array}$} \\
\hline $\begin{array}{l}\text { Recurrent depression } \\
\text { Index depression coded as }\end{array}$ & $815(9.70)$ & $1964(8.05)$ & $99(8.85)$ & 2335 (13.58) & $160(12.89)$ & N/A \\
\hline hospital discharge diagnosis & $191(2.27)$ & $496(2.03)$ & $24(2.14)$ & $647(3.76)$ & $46(3.71)$ & N/A \\
\hline
\end{tabular}




\begin{tabular}{|c|c|c|c|c|c|c|}
\hline & $\begin{array}{l}\text { SNRI group } \\
(n=8403)\end{array}$ & $\begin{array}{l}\text { SSRI group } \\
(n=24398)\end{array}$ & $\begin{array}{l}\text { TCA group } \\
(n=1119)\end{array}$ & $\begin{array}{c}\text { Multiple } \\
\text { antidepressants } \\
\text { group }(n=17 \text { 194) }\end{array}$ & $\begin{array}{l}\text { Untreated } \\
\text { depression group } \\
(n=1241)\end{array}$ & $\begin{array}{l}\text { General } \\
\text { population sample } \\
(n=75756)\end{array}$ \\
\hline $\begin{array}{l}\text { Chronic Disease Indicator } \\
\text { score }^{21} \text { mean (median) } \\
\text { Range, minimum-maximum }\end{array}$ & $\begin{array}{l}2.66(2.00) \\
0-21\end{array}$ & $\begin{array}{l}2.82(2.00) \\
1-21\end{array}$ & $\begin{array}{l}4.71(4.00) \\
2-18\end{array}$ & $\begin{array}{l}3.30(3.00) \\
0-19\end{array}$ & $\begin{array}{l}1.52(0.00) \\
0-13\end{array}$ & $\begin{array}{l}1.56(1.00) \\
0-22\end{array}$ \\
\hline $\begin{array}{l}\text { Charlson comorbidity score, } \\
\text { mean (median) } \\
\text { Range, minimum-maximum }\end{array}$ & $\begin{array}{l}0.71(0.00) \\
0-10\end{array}$ & $\begin{array}{l}0.71(0.00) \\
0-14\end{array}$ & $\begin{array}{l}0.94(1.00) \\
0-9\end{array}$ & $\begin{array}{l}0.74(0.00) \\
0-11\end{array}$ & $\begin{array}{l}1.17(1.00) \\
0-12\end{array}$ & $\begin{array}{l}0.00(0.00) \\
0-0\end{array}$ \\
\hline Prior depression, $n(\%)$ & $0(0.00)$ & $0(0.00)$ & $0(0.00)$ & $0(0.00)$ & $0(0.00)$ & $1964(2.59)$ \\
\hline Bipolar disorder, $n$ (\%) & $34(0.40)$ & $78(0.32)$ & $8(0.71)$ & $80(0.47)$ & $24(1.93)$ & $382(0.50)$ \\
\hline Schizophrenia, $n$ (\%) & $6(0.07)$ & $19(0.08)$ & $1(0.09)$ & $5(0.03)$ & $19(1.53)$ & $51(0.07)$ \\
\hline Psychotic disorder, $n$ (\%) & $47(0.56)$ & $147(0.60)$ & $7(0.63)$ & $120(0.70)$ & $19(1.53)$ & $174(0.23)$ \\
\hline Anxiety spectrum disorder, $n$ (\%) & $588(7.00)$ & $1624(6.66)$ & $61(5.45)$ & $1187(6.90)$ & 99 (7.98) & $1713(2.26)$ \\
\hline $\begin{array}{l}\text { Attention-deficit hyperactivity } \\
\text { disorder, } n(\%)\end{array}$ & $44(0.52)$ & $130(0.53)$ & $0(0.00)$ & $76(0.44)$ & $7(0.56)$ & $325(0.43)$ \\
\hline Substance use disorder, $n(\%)$ & $320(3.81)$ & $869(3.56)$ & $51(4.56)$ & $791(4.60)$ & $70(5.64)$ & $1438(1.90)$ \\
\hline Other mental health disorder, $n$ (\%) & 307 (3.65) & $864(3.54)$ & $47(4.20)$ & $696(4.05)$ & $66(5.32)$ & $1877(2.48)$ \\
\hline $\begin{array}{l}\text { Number of mental health disorders, } \\
\text { mean (median) } \\
\text { Range, minimum-maximum }\end{array}$ & $\begin{array}{l}0.16(0.00) \\
0-5\end{array}$ & $\begin{array}{l}0.15(0.00) \\
0-5\end{array}$ & $\begin{array}{l}0.15(0.00) \\
0-4\end{array}$ & $\begin{array}{l}0.17(0.00) \\
0-5\end{array}$ & $\begin{array}{l}0.24(0.00) \\
0-5\end{array}$ & $\begin{array}{l}0.08(0.00) \\
0-5\end{array}$ \\
\hline $\begin{array}{l}\text { Terminal diagnosis (HIV or malignant } \\
\text { neoplasm), } n(\%)\end{array}$ & $233(2.77)$ & $688(2.82)$ & $45(4.02)$ & $434(2.52)$ & $57(4.59)$ & $1436(1.90)$ \\
\hline Smoking, $n(\%)$ & $28(0.33)$ & $86(0.35)$ & $5(0.45)$ & $55(0.32)$ & $8(0.64)$ & $119(0.16)$ \\
\hline Seizure disorder, $n$ (\%) & $54(0.64)$ & $157(0.64)$ & $13(1.16)$ & $90(0.52)$ & $20(1.61)$ & $265(0.35)$ \\
\hline Chronic pain, $n(\%)$ & $16(0.19)$ & $30(0.12)$ & $1(0.09)$ & $14(0.08)$ & $19(1.53)$ & $41(0.05)$ \\
\hline Headaches (including migraine), $n$ (\%) & $579(6.89)$ & $1533(6.28)$ & $170(15.19)$ & $1340(7.79)$ & $98(7.90)$ & $3123(4.12)$ \\
\hline Stress urinary incontinence, $n$ (\%) & $38(0.45)$ & $102(0.42)$ & $4(0.36)$ & $76(0.44)$ & $12(0.97)$ & $223(0.29)$ \\
\hline Fibromyalgia, $n(\%)$ & $325(3.87)$ & 837 (3.43) & $62(5.54)$ & 677 (3.94) & $87(7.01)$ & $1491(1.97)$ \\
\hline Diabetic neuropathy, $n$ (\%) & $206(2.45)$ & $559(2.29)$ & $51(4.56)$ & $447(2.60)$ & $62(5.00)$ & 1005 (1.33) \\
\hline Low back pain/back pain, $n$ (\%) & 959 (11.41) & $2567(10.52)$ & $173(15.46)$ & $2194(12.76)$ & 220 (17.73) & 4616 (6.09) \\
\hline
\end{tabular}

score matching to illustrate both the need for and the effect of the propensity score matching process, based on the demographic and clinical characteristics of the study groups). As seen in Table 1, the study cohorts are comparable on factors associated with suicide risk after propensity matching. Mean age of study participants varied only from 41 to 44 years. Roughly two-thirds of each cohort were women, as would be expected in samples of adults with depression; the proportion of women was similar in the general population sample because of age and gender matching. Based on the exclusion criteria used to create the cohorts, by definition, there was no prior depression in the depression groups during the 12 months prior to the index episode; $2.6 \%$ of the general population sample had a depression diagnosis during the 6 months prior to their index date. The most common comorbidity (Table 2) seen prior to the index date was anxiety spectrum disorder (5-8\% in the depression groups, $2 \%$ in the general population sample). Prevalence of prior suicide attempt was low (Table 1), ranging from $0.18 \%$ to $0.62 \%$ in the depression groups and only $0.01 \%$ in the general population sample. The index depression diagnosis indicated recurrent illness for $8-14 \%$ of patients (Table 1).

Monthly aggregated suicide attempt rates in relation to index date are presented for all ages in Fig. 2. For all the treated depression groups (SNRI, SSRI, TCA and multiple antidepressants), suicide attempt rates were highest in the month before diagnosis and treatment initiation, and steadily decreased over the next 6 months. The highest rate for the untreated depression group was seen in the second month following the depression diagnosis. Although the low rate for the general population sample was difficult to determine from the figure, it was higher 6 months prior to the index date (5.3 per 100000) and 3 months following the index date $(4.2$ per 100000$)$ and displayed much less of a clear pattern or trend over time, as would have been expected.

Figure 3 displays suicide attempt rates for the follow-up period by age group. Young adult and adult groups are shown; there were no suicide attempts to display in the older adult age group. Treatment cohort crude suicide attempt rates are presented as absolute (attributable) risk differences from the general population sample community-based cohort. The multiple antidepressants group had the highest suicide attempt rate, with an absolute risk difference (ARD) of 2156 additional attempts per 100000 and 498 additional attempts per 100000 in the young adult and adult cohorts, respectively, compared with the general population sample. In the adult age group (ages 25-64), the untreated depression group had statistically significantly higher suicide attempt rates (ARD of 163 additional attempts per 100000 ) relative to the general population sample, an ARD that was higher than those observed for the SNRI, SSRI or TCA treated groups (ARDs of 113,81 and 79 additional attempts per 100000 respectively). In the young adult (age 19-24) age group, no suicide attempts were observed for participants in the untreated depression or TCA groups, resulting in ARDs of 82 attempts per 100000 lower (less) than the general population sample, although the rarity of events in these two specific groups makes the estimates less precise.

Table 3 presents multivariate Cox proportional hazard model results on time to suicide attempt across the study groups, stratified 


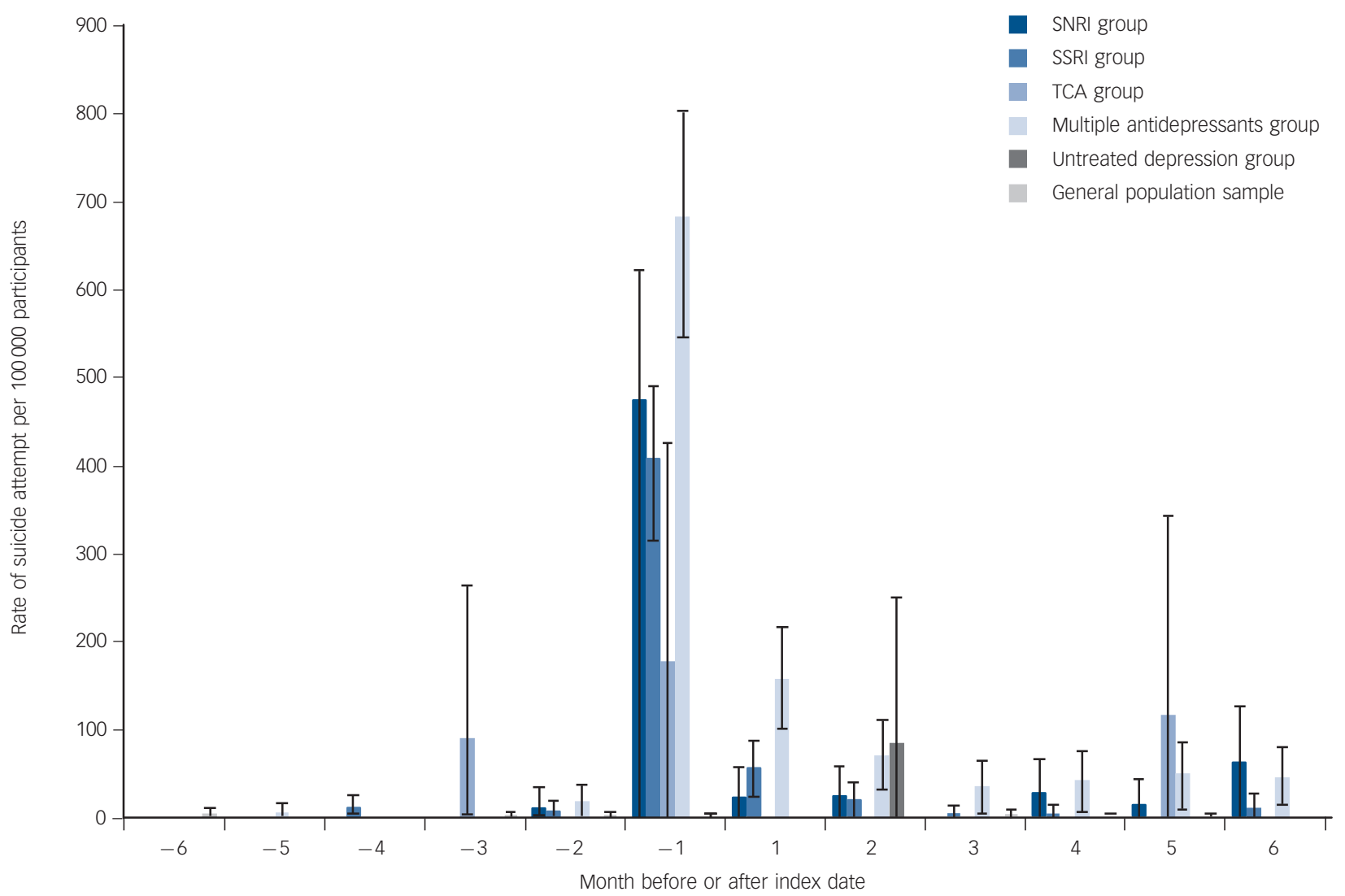

Fig. 2 Crude rates of suicide attempt during the 6 months prior to and following the index antidepressant prescription date, all age groups aggregated.

Suicide attempts occurring on the index date were included in the prior month. Standard errors shown by whiskers. SNRI, serotonin-noradrenaline reuptake inhibitor; SSRI, selective serotonin reuptake inhibitor; TCA, tricyclic antidepressant.

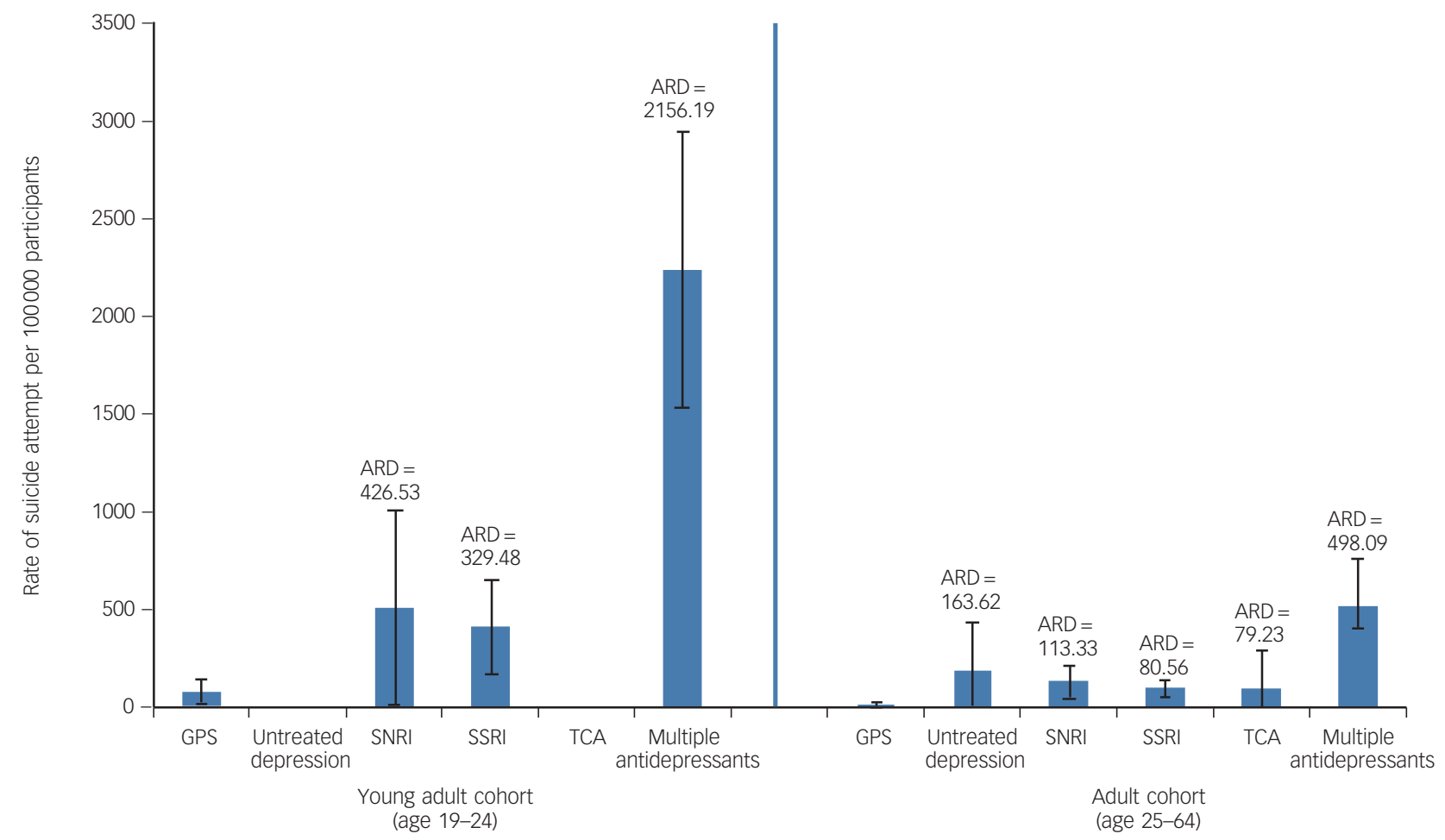

Fig. 3 Rate of suicide attempt and absolute risk difference (ARD) for depression cohorts relative to the general population sample (GPS) cohort, by age group.

No results are presented for the older adult age cohort because there were no suicide attempts in that group. Whiskers show $95 \%$ confidence intervals. SNRI, serotonin-noradrenaline reuptake inhibitor; SSRI, selective serotonin reuptake inhibitor; TCA, tricyclic antidepressant. 


\begin{tabular}{|c|c|c|}
\hline \multirow[b]{2}{*}{ Group } & \multicolumn{2}{|c|}{ Hazard ratio $(95 \% \mathrm{Cl})$} \\
\hline & Young adult, age 19-24 ( $n=12673)$ & Adult, age 25-64 $(n=113710)$ \\
\hline \multicolumn{3}{|l|}{ Referent group, general population sample } \\
\hline Serotonin-noradrenaline reuptake inhibitor & $7.62(1.99-29.20)$ & $9.00(3.60-22.50)$ \\
\hline Selective serotonin reuptake inhibitor & $4.51(1.50-13.59)$ & $6.87(3.10-15.20)$ \\
\hline Tricyclic antidepressant & NE & $5.10(0.64-40.84)$ \\
\hline Multiple antidepressants & $20.92(7.81-56.04)$ & $23.87(11.70-48.73)$ \\
\hline Untreated depression & NE & $10.65(2.11-53.80)$ \\
\hline \multicolumn{3}{|l|}{ Referent group, untreated depression group } \\
\hline Serotonin-noradrenaline reuptake inhibitor & NE & $0.85(0.17-4.19)$ \\
\hline Selective serotonin reuptake inhibitor & NE & $0.65(0.14-3.02)$ \\
\hline Tricyclic antidepressant & NE & $0.48(0.04-5.65)$ \\
\hline Multiple antidepressants & NE & $2.24(0.50-10.02)$ \\
\hline General population sample & NE & $0.09(0.02-0.46)$ \\
\hline \multicolumn{3}{|c|}{ Referent group, serotonin-noradrenaline reuptake inhibitor } \\
\hline Selective serotonin reuptake inhibitor & $0.59(0.19-1.90)$ & $0.76(0.36-1.63)$ \\
\hline Tricyclic antidepressant & NE & $0.57(0.07-4.46)$ \\
\hline Multiple antidepressants & $2.75(0.97-7.82)$ & $2.65(1.63-5.16)$ \\
\hline Untreated depression & NE & $1.18(0.24-5.86)$ \\
\hline General population sample & $0.13(0.03-0.50)$ & $0.11(0.04-0.28)$ \\
\hline
\end{tabular}

by age group (again, without reporting older adults because of no events). The association of treatment drug class on suicide risk was estimated fully adjusting for propensity to receive an SNRI, comorbidities, drug-months of exposure to prior prescriptions and suicide attempt history, among other relevant variables (listed in Tables 1 and 2). Three separate multivariate models were estimated for young adults and adults; each model varied the reference group (SNRI, untreated depression or general population sample) in order to more fully describe the relative associations between different classes of drug treatments and suicide risk. The top panel reports hazard ratios (HR) with the general population sample as the referent group. The depression cohorts, both treated and untreated, had significantly higher risk for suicide attempt compared with the general population, with a greater magnitude for adults over age 24 than for young adults. Indeed, comparison with the general population sample as a referent group shows the statistically significant association of depression with suicide risk present in the study cohorts.

The middle panel reports hazard ratios using the untreated depression group as the referent group; antidepressant-treated groups were not significantly different from the untreated depression group on the relative hazard of suicide attempt. These data are consistent with the illness of depression as the major driver of suicide attempts, with rates decreasing on a population basis after antidepressant treatment is initiated. Nonetheless, population data do not fully address the risk of suicidal behaviour that has been reported in placebo-controlled randomised trials in some populations (i.e. adolescents). As such, clinical monitoring for suicide risk in at-risk populations is still prudent.

The bottom panel reports results with the SNRI group as referent group in order to examine suicide attempt risk associated with specific classes/types of antidepressants. There was no significant difference in suicide attempt risk between groups treated with SNRIs and SSRIs. There were only two SNRIs included in the analysis - duloxetine and venlafaxine - and they were examined as individual agents as well as an SNRI class (individual agent results provided in online Table DS7). When compared with patients treated with duloxetine (Table DS7), patients treated with venlafaxine $(\mathrm{HR}=1.01,95 \% \mathrm{CI} 0.6-1.8)$ or
SSRIs $\quad(\mathrm{HR}=0.89,95 \%$ CI $0.5-1.5)$ showed no statistically different rate of suicide attempt. The multiple antidepressants group had significantly increased risk of suicide attempt compared with the duloxetine subgroup ( $\mathrm{HR}=3.11,95 \% \mathrm{CI} 1.9-5.0)$.

\section{Discussion}

\section{Main findings}

This study presents the most complete set of comparative risk estimates published to date regarding the risk of suicide attempt associated with antidepressant treatment in adults during naturalistic treatment. In addition to antidepressant drug class $v$. class comparisons, we included a general population cohort that permitted estimation of the association between the underlying illness (depression) and suicide attempt. In the Cox proportional hazard models, the depression cohort, whether treated or untreated, had significantly higher risk for suicide attempt compared with the general population. The comparison with an untreated depression group further supports the hypothesis that depressive illness underlies the risk of suicide attempt observed among the antidepressant-treated groups. In fact, in comparison with the untreated depression group, patients taking antidepressants of any individual class did not have a significantly higher risk of suicide attempt. Only users of multiple antidepressant classes (either concurrently or consecutively) had higher risk of suicide attempt.

It might have been hypothesised that patients in the depression cohort who were treated would have had lower rates of suicide attempt than those individuals who were not. In reality, however, the lack of pharmacological treatment in the untreated depression group may have reflected a judgement by the prescribing physician that the patient's depression was mild and/ or that the perceived risk of suicide or other adverse outcomes was low, and that pharmacological treatment was therefore not required. Conversely, given that TCAs are increasingly reserved for more severely ill patients, a higher rate of suicide attempts might have been expected in this cohort, but we did not see this. The relatively low rate of suicide attempts in this group may reflect the fact that TCAs are highly toxic and at times lethal in overdose 
relative to SNRIs and SSRIs, ${ }^{24}$ and as a consequence prescribers may avoid their use in patients considered to be at a risk of suicide. The risk of suicide attempt in the multiple antidepressants group was significantly higher than in other groups, perhaps reflecting a harder to treat patient population or a situation in which the use of multiple antidepressant classes might carry additional unknown risks.

\section{SNRIS}

Considering the SNRIs, there are only two individual agents in this class. They were examined both individually and combined, with no differences in results, thus providing the first results for the latest SNRI on the market, duloxetine. Contrary to some previous reports, ${ }^{8,25}$ our study did not suggest the existence of a significantly higher suicide attempt risk among patients using SNRIs as a class compared with those using SSRIs. It may be that the SNRIs are reserved by prescribers for the treatment of patients with severe or chronic depression or whose depression is treatmentresistant compared with those prescribed SSRIs, so the SNRI-treated group may represent one at higher risk of suicide attempt. ${ }^{26}$ Some prior studies have not controlled for baseline differences (such as prior suicide attempt history), so more suicide attempts would be expected in patients receiving SNRIs $v$. SSRIs. The fact that our study controlled for many baseline differences makes the study important, and may at least partially explain the lack of any observed differences in risk of suicide attempt between the SNRI and SSRI cohorts. Likewise, no differences among individual SSRI subgroups were detected (in comparison with either fluoxetine as a referent SSRI, or the TCA, multiple antidepressants or the untreated depression groups; results not shown, because of lack of observed differences between groups).

\section{Strengths and limitations}

The cohort creation and multivariate methods used in this study represent the state of the art in accounting for non-random allocation to treatment and the associated confounding that might be observed in a treatment-outcome relationship. ${ }^{27}$ We used the standard approach of creating an incident depression cohort with the associated clean sweep period. We then were able to utilise the large sample size in our database to create propensity-matched cohorts. Finally, we used further multivariate adjustment using measures of known, measurable risk factors in claims data for later suicide attempt (such as history of suicide attempt, prior depression episode) to further account for residual confounding. Our results are consistent with prior work showing an elevation in risk of suicide attempt prior to initiation of antidepressant therapy, and a gradual reduction in risk over time after initiation. ${ }^{13,28}$

Limitations to this study should be taken into account when interpreting the findings. Data for the study were drawn from integrated health insurance claims records, which are subject to underreporting of diagnosis because of stigma or financial disincentives, to underreporting of antidepressant prescriptions because of the use of samples and to underreporting of suicide attempts that may not reach the level of seriousness to result in a medical claim, but may nevertheless be real. These limitations may potentially bias our estimates in a downward direction, leaving the results as a low estimate of the statistical parameter. There is no reason, however, to expect that any of the possible underreporting would bias any one study group or finding in a particular direction, or bias across-group (relative risk) comparisons. Second, our study does not capture completed suicides, aborted attempts or suicidal thoughts or ideation. Thus, only one dimension of suicide-related behaviour is addressed, and our findings do not generalise to other dimensions of this complex phenomenon. Further, the use of propensity score matching, as intended, 'levelled the playing field' by making the study drug groups more comparable in terms of many demographic and clinical variables, but there were still some residual imbalances between the groups (such as rates of recurrent depression were higher in the untreated depression group and multiple antidepressants group than the single antidepressant groups). In addition, propensity score matching can only match on the basis of identified, measured confounders and there may be other confounders that are either unknown or unmeasured, that contribute to residual differences between groups. Finally, we did not study child or adolescent populations, having addressed them in prior work, ${ }^{28}$ so our findings are generalisable only to adult populations.

In summary, although focusing on the risk of suicide attempt among users of SNRI antidepressants, this study adds risk estimates to the literature on key, previously unmeasured cohorts: the general population, with depression but pharmacologically untreated and the SSRI, SNRI and multiple antidepressants groups. We also contribute new data in regard to the most recent SNRI introduced to the market. Large, retrospective new-user cohorts are the ideal study design for such work because of the need to detect not only the rare outcome of suicide attempt, but also to create statistically matched cohorts. These estimates are readily interpretable for such metrics as number needed to harm and likelihood of help and harm because they were derived from real-world effectiveness samples from a broad-based US managed care population. Future work will follow up on this analysis with further investigation into the complexity of describing drug regimens and measuring suicide-related behaviours and events among users of multiple antidepressants, and the study of multiple suicide attempts over time.

Robert J. Valuck, PhD, RPh, FNAP, Department of Clinical Pharmacy, University of Colorado Skaggs School of Pharmacy and Pharmaceutical Sciences, Aurora,

Colorado, USA; Anne M. Libby, PhD, Department of Emergency Medicine, University of Colorado School of Medicine, Aurora, Colorado, USA; Heather D. Anderson, PhD, Department of Clinical Pharmacy, University of Colorado Skaggs School of Pharmacy and Pharmaceutical Sciences, Aurora, Colorado, USA; Richard R. Allen, MS, Peak Statistical Services, Evergreen, Colorado, USA; Indiana Strombom, PhD, Genentech, San Francisco, California, USA; Lauren B. Marangell, MD, Department of Psychiatry, University of Texas Health Science Center, Houston, Texas, and Brain Health Consultants, Houston, Texas, USA; David Perahia, MBBS, BSC, MRCPsych, Eli Lilly and Company, Erl Wood, Windlesham, UK

Correspondence: Dr David G. S. Perahia, Lilly Research Centre, Erl Wood Sunninghill Road, Windlesham, Surrey GU20 6PH, UK. Email: perahia_david_g@ lilly.com

First received 5 May 2014, final revision 8 Apr 2015, accepted 14 Apr 2015

\section{Funding}

This study was funded by Eli Lilly and Company, Indianapolis, per a regulatory study request by the European Medicines Agency (EMA).

\section{References}

1 Mayer-Gross W, Slater E, Roth M. Clinical Psychiatry. Bailliere, Tindell and Cassell, 1960

2 Hammad TA, Laughren T, Racoosin J. Suicidality in pediatric patients treated with antidepressant drugs. Arch Gen Psychiatry 2006; 63: 332-9.

3 Mosholder $A D$, Pamer CA. Postmarketing surveillance of suicidal adverse events with pediatric use of antidepressants. J Child Adolesc Psychopharmacol 2006; 16: 33-6.

4 Tollefson GD. Fluoxetine and suicidal ideation. Am J Psychiatry 1990; 147: 1691-3.

5 Libby AM, Orton HD, Valuck RJ. Persisting decline in depression treatment after FDA warnings. Arch Gen Psychiatry 2009; 66: 633-9. 
6 Libby AM, Brent DA, Morrato EH, Orton HD, Allen R, Valuck RJ. Decline in treatment of pediatric depression after FDA advisory on risk of suicidality with SSRIs. Am J Psychiatry 2007; 164: 884-91.

7 Valuck RJ, Libby AM, Orton HD, Morrato EH, Allen R, Baldessarini RJ. Spillover effects on treatment of adult depression in primary care after FDA advisory on risk of pediatric suicidality with SSRIs. Am J Psychiatry 2007; 164: 1198-205.

8 Medicines and Healthcare Products Regulatory Agency (MHRA). Committee on Safety of Medicines Assessment Report: Seroxat (Paroxetine) - Safety and Efficacy in the Paediatric Population. MHRA, 2003 (http://webarchive. nationalarchives.gov.uk/20141205150130/http://www.mhra.gov.uk/home/ groups/es-policy/documents/websiteresources/con014159.pdf ).

9 Food and Drug Administration. Overview for December 13 Meeting of Psychopharmacologic Drugs Advisory Committee (PDAC). FDA, 2006 (http:// www.fda.gov/ohrms/dockets/ac/06/briefing/2006-4272b1-01-FDA.pdf).

10 Hammad TA, Laughren TP, Racoosin JA. Suicide rates in short-term randomized controlled trials of newer antidepressants. J Clin Psychopharmacol 2006; 26: 203-7.

11 Saperia J, Ashby D, Gunnell D. Suicidal behaviour and SSRIs: updated metaanalysis. BMJ 2006; 332: 1453.

12 Gunnell D, Saperia J, Ashby D. Selective serotonin reuptake inhibitors (SSRIS) and suicide in adults: meta-analysis of drug company data from placebo controlled, randomised controlled trials submitted to the MHRA's safety review. BMJ 2005; 330: 385

13 Simon GE, Savarino J, Operskalski B, Wang PS. Suicide risk during antidepressant treatment. Am J Psychiatry 2006; 163: 41-7.

14 Martinez C, Rietbrock S, Wise L, Ashby D, Chick R, Moseley J, et al. Antidepressant treatment and the risk of fatal and non-fatal self harm in first episode depression: nested case-control study. BMJ 2005; 330: 389.

15 Jick $\mathrm{H}$, Kaye JA, Jick SS. Antidepressants and the risk of suicidal behaviors. JAMA 2004; 292: 338-43.

16 Schneeweiss S, Patrick AR, Solomon DH, Mehta J, Dormuth C, Miller M, et al. Variation in the risk of suicide attempts and completed suicides by antidepressant agent in adults: a propensity score-adjusted analysis of 9 years' data. Arch Gen Psychiatry 2010; 67: 497-506.

17 IMS Health. IMS LifeLink ${ }^{T M}$ Health Plan Claims Data User Guide \& Data Dictionary. IMS Health, 2011.
18 World Health Organization. The ICD-10 Classification of Mental and Behavioural Disorders: Clinical Descriptions and Diagnostic Guidelines. WHO, 1992.

19 Centers for Disease Control and Prevention. Injury Prevention \& Control: Data \& Statistics (WISQARS ${ }^{T M}$ ). Table 1. Recommended Framework of E-code Groupings for Presenting Injury Mortality and Morbidity Data (August 10, 2011). CDC, 2011 (http://www.cdc.gov/injury/wisqars/ecode_matrix.html)

20 Centers for Disease Control and Prevention. Tools for Classifying ICD Codes: External Cause-of-Injury (E-code) Matrices. Codes for the Matrix. Centers for Disease Control and Prevention, 2011. Available at: http://www.cdc.gov/ nchs/injury/injury_tools.htm (accessed 20 Oct 2015).

21 Malone D, Billups S, Valuck R, Carter BL. Development of a chronic disease indicator score using a Veterans Affairs Medical Center medication database. J Clin Epidemiol 1999; 52: 551-7.

22 D'Hoore W, Bouckaert A, Tilquin C. Practical considerations on the use of the Charlson comorbidity index with administrative data bases. J Clin Epidemiol 1996; 49: 1429-33.

23 Cleophas TJ, Zwinderman AH. Propensity Score Matching. Statistics Applied to Clinical Studies. Springer Netherlands, 2012.

24 Whyte IM, Dawson AH, Buckley NA. Relative toxicity of venlafaxine and selective serotonin reuptake inhibitors in overdose compared to tricyclic antidepressants. QJM 2003; 96: 369-74.

25 Rubino A, Roskell N, Tennis P, Mines D, Weich S, Andrews E. Risk of suicide during treatment with venlafaxine, citalopram, fluoxetine, and dothiepin: retrospective cohort study. BMJ 2007; 334: 242.

26 Mines D, Hill D, Yu H, Novelli L. Prevalence of risk factors for suicide in patients prescribed venlafaxine, fluoxetine, and citalopram. Pharmacoepidemiol Drug Saf 2005; 14: 367-72.

27 Velentgas $\mathrm{P}$, Dreyer NA, Nourjah P, Smith SR, Torchia MM. Developing a Protocol for Observational Comparative Effectiveness Research: A User's Guide. AHRQ Publication No. 12(13)-EHC099. Agency for Healthcare Research and Quality, 2013.

28 Valuck RJ, Libby AM, Sills MR, Giese AA, Allen RR. Antidepressant treatment and risk of suicide attempt by adolescents with major depressive disorder: a propensity-adjusted retrospective cohort study. CNS Drugs 2004; 18 : 1119-32. 